\title{
A MONTE CARLO STUDY OF NEUTRON THERMALIZATION*
}

by

\author{
H. D. Brown
}

Savannah River Laboratory

E. I. du Pont de Nemours \& Co. Alken, South Carolina

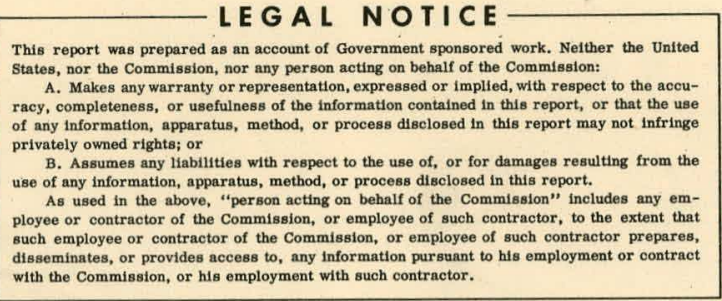

* The information contained in this report was developed during the course of work under contract AT(07-2)-1 with the Atomic Energy Commission, whose permission to publish is gratefully acknowledged. 


\section{DISCLAIMER}

This report was prepared as an account of work sponsored by an agency of the United States Government. Neither the United States Government nor any agency Thereof, nor any of their employees, makes any warranty, express or implied, or assumes any legal liability or responsibility for the accuracy, completeness, or usefulness of any information, apparatus, product, or process disclosed, or represents that its use would not infringe privately owned rights. Reference herein to any specific commercial product, process, or service by trade name, trademark, manufacturer, or otherwise does not necessarily constitute or imply its endorsement, recommendation, or favoring by the United States Government or any agency thereof. The views and opinions of authors expressed herein do not necessarily state or reflect those of the United States Government or any agency thereof. 


\section{DISCLAIMER}

Portions of this document may be illegible in electronic image products. Images are produced from the best available original document. 


\title{
A MONTE CARLO STUDY OF NEUTRON THERMALIZATION: \\ by H. D. Brown
}

\begin{abstract}
The slowing down of neutrons from fission to absorption energies in a homogeneous medium has been treated by a Monte Carlo method. Thermalization by elastic collisions in both light and heavy water was computed on an IBM 650. One-s1xth the mean-square distance travelled by the neutrons was interpreted as the migration area. This quantity was analyzed to give the age, $\tau$, both above and below the Indium resonance, and the thermal diffusion constant, $D$, as functions of the moderator temperature, the effective moderator mass, the moderator composition, the scattering and absorption laws, and the energy distribution of the neutron source.

The migration area from the fission energy source to below the indium resonance energy $(1.46 \mathrm{ev})$ was found to be $27.4 \mathrm{~cm}^{2}$ for $\mathrm{H}_{2} \mathrm{O}$ and $120 \mathrm{~cm}^{2}$ for $\mathrm{D}_{2} \mathrm{O}$. By varying the absorption cross section, the migration area from the indium resonance energy to absorption of the neutrons could be decomposed into components $D / L^{2}$ and $\tau_{s} \cdot D$ and $\tau_{s}$ were found to be. $0.19 \mathrm{~cm}$ and $0.77 \mathrm{~cm}^{2}$ respectively for $\mathrm{H}_{2} \mathrm{O}$, and $0.87 \mathrm{~cm}$ and $20 \mathrm{~cm}^{2}$ for $\mathrm{D}_{2} \mathrm{O}$. D was found to increase with the thermal agitation of the moderator at the rate of $0.15 \% /{ }^{\circ} \mathrm{C}$ for $\mathrm{H}_{2} \mathrm{O}$ and $0.14 \% /{ }^{\circ} \mathrm{C}$ for $\mathrm{D}_{2} \mathrm{O}$. Neutron energy distributions found from the scattering frequency agree with those derived by analytical methods. (auth)
\end{abstract}




\section{A MONTE CARLO STUDY OF NEUTRON THERMAIIZATION}

by H. D. Brown

\section{INTRODUCTION}

A comprehensive study of neutron thermalization involves the diffusion of neutrons in space and energy as they are slowed from fission energies to thermal absorption. Such a study must be concerned with the energy of the source of neutrons, the scattering and absorption cross sections over the entire slowing-down range, the molecular mass of the moderator, 1ts temperature, and the molecular binding.

The solution of the thermalization problem is needed to compute moderator properties such as migration areas and group constants. These in turn are used to estimate $k$ eff for reactors, for subcritical assemblies, and for containers of fissile materials. Further objectives of the calculation were to study the decomposition of the migration area into fast and thermal components, and to find the variation of the migration area with the temperature of the moderator.

Other slowing-down studies have been based on analytical evaluation of the moments of the slowing-down distribution (Wilkins, Hellens, and Zweifel (1956)). However, variation of parameters by analytical methods is difficult because of the complexity of the solutions. A question unresolved by the earlier work was the effect of temperature on the slowing-down phenomenon in the transition region between fast and slow neutrons. Parametric variation is particularly easy in Monte Carlo methods where the input data can have arbitrary form. 


\section{THE MONTE CARLO CODE}

A Monte Carlo code for the IBM 650 computer is used at the Savannah River Laboratory to compute slowing down in an infinite homogeneous medium. Neutron histories are computed, collision by collision, as the neutrons are thermalized from the fission source. In each collision elther a scattering or an absorption takes place according to the relative cross section at the neutron's energy. If the neutron is absorbed a new history is begun. If it is scattered, the energy loss in scattering is determined from the equations of elastic collision. It is assumed that the scattering is isotropic in the center-of-mass frame of reference. After scattering, a new event occurs, again as determined by the relative cross sections for scattering and absorption. This process is continued until the neutron is absorbed or until it is slowed down past the range of interest. The calculation was performed in two parts; slowing down from fission to Indium resonance $(1.46 \mathrm{ev}$ ) and slowing down from indium resonance to absorption. A $I / v$ absorption rate was assumed in the energy range below the indium resonance, but the code is not restricted to such an assumption.

Precision 1s improved and the computing time speeded by a statistical estimation of the migration area and through the extensive use of tables. Cross sections, source energy distributions, and moderator energy distributions are obtained, as needed, from tables instead of being computed from formulas. Eight digit random numbers, $R$, were generated by a process suggested by Lehmer (1951) which is well sulted for application to digital computers: 


$$
\mathrm{R}_{\mathrm{n}+1}=23 \mathrm{R}_{\mathrm{n}} \bmod \left(10^{8}+1\right)
$$

The random numbers are used to choose scattering and absorption probabilities, angles of scattering, and target velocities.

A neutron history is acquired in the following sequence:

a) select the initial neutron energy, b) determine the type of event., c) assign the scattering mass, d) determine the target velocity, e) calculate the energy loss, f) accumulate the addition to the migration area, then begin the next event. When a neutron Is absorbed or scattered out of the energy range of interest, the history is terminated. These steps are discussed in the following paragraphs.

Initial Energy

The initial energy may be the same for all neutrons as it is for slowing down from indium resonance to absorption, or it may be taken in tabulated order from a properly biased table as it is for high energy calculations. The source for high energy scattering was taken to be a fission energy distribution in the form of a least-squares maxwellian fit to data reported by Rosen (1955). Sequential sampling of the entire table assures an accurate representation.

Type of Event

The probability, $p_{1}$, of an event of type 1 occurring among competing events is $p_{1}=\frac{\sigma_{1}}{\Sigma_{j} \sigma_{j}}$, where the $\sigma_{j}$ are macroscopic cross sections in the laboratory frame of reference. Tables of $P_{1}=\sum_{k=1}^{1} p_{k}$ are stored in the IBM 650 as functions of energy. An event is of type 1 if a number $R$, selected at random from the range 0 to 1 , is in the interval $P_{1-1} \leqslant R<P_{1}$. If the event is an absorption, the migration area at which absorption occurs, the 
energy at which absorption occurs, the nuclear identity of the absorbing atom, the number of the neutron, and the number of the collision are recorded. A new neutron is then selected from the source.

Scattering Mass

Chemical binding of the moderator (target) atoms, such as that encountered in water moderators, increases the inertia of the target in collision. The influence of this effect is approximated in the calculation by using a larger mass for the target atom.

When the incident neutron has energy in excess of the vibrational binding energy of the molecule, $E^{*}$, it is assumed that the target is unbound and free atom masses are used. When the energy of the incident neutron is less than $E^{*}$, the effective masses derived by Brown and St. John (1954) are employed. Thus, In $\mathrm{D}_{2} \mathrm{O}$, the effective mass of oxygen, $\mathrm{A}(\mathrm{O})$, is 16 and that of deuterium, $A(D)$ is 2 for events occurring above $E^{*}=0.143 \mathrm{ev}$. $A(O)$ is 20 and $A(D)$ is 3.6 for events occurring below $E^{*}$. Similarly for $\mathrm{H}_{2} \mathrm{O}, \mathrm{A}(\mathrm{O})$ is 18 and $\mathrm{A}(\mathrm{H})$ is 1.9 for events occurring below $E^{*}=0.226 \mathrm{ev}$.

\section{Target Velocity}

The probability, $p$, of collision between a neutron of velocity $\vec{u}$ and a target of velocity $\vec{U}$ depends upon the relative velocity and the thermal distribution of the target atom velocities. It is customary to assume that the target atoms possess a maxwellian distribution of velocities, $M(\vec{U})$, in the laboratory system and that the scattering cross section in the center-of-mass coordinates is independent of the target velocity. The probability of collision 
with a moderator atom is obtained by integrating over all possible velocities.

$$
\begin{gathered}
\int p d \vec{U}=\int_{0}^{\infty}|\vec{u}-\vec{U}| M(\vec{U}) d \vec{U} \\
=\frac{1}{\beta \sqrt{\pi}} e^{-\beta^{2} u^{2}}+\left(\frac{1}{2 \beta^{2} u}+u\right) \operatorname{Erf}(\beta u)
\end{gathered}
$$

where $\beta^{2}=\frac{A}{2 k T}, k T$ represents the moderator temperature, and $\operatorname{Erf}(x)=\frac{2}{\sqrt{\pi}} \int_{0}^{x} e^{-y^{2}} d y$ is the error function. The target velocity must be selected from equally weighted samples of this integral probability.

Since the integral probability is complex and difficult to decompose into equally weighted samples, we deal instead with the differential probability. The selection of $U$ is performed by a rejection technique. An upper bound of the integrand is $u+U$. Thus, an upper bound of the probability is

$$
\begin{gathered}
\int p_{\max } d \vec{U}=u \int_{0}^{\infty} M(U) d U+\int_{0}^{\infty} U M(U) d U \\
\quad \equiv u+I
\end{gathered}
$$

A random number $R_{1}$ is drawn to decide the selection of $U$. If $R_{1} \leqslant \frac{u}{u+I}$, a value of $U$ is taken at random from a table blased with a maxwellian distribution. If $R_{1}>\frac{u}{u+I}, U$ is selected from a table biased with a U-weighted maxwellian distribution. Although the $U$ so obtained is representative of the upper bound probability, $\mathrm{p}_{\max }$, the actual probability is more restrictive. Every velocity $U$ is chosen too frequently by the $p_{\max }$ sampling. The frequency should be reduced by the fraction by which $p_{\max }$ exceeds the actual $p$. Thus a second random number, $R_{2}$, is drawn to test the cholce of $U$. If $R_{2} \leqslant \frac{\vec{u}-\vec{U}}{u+U}, U$ is accepted as the target velocity. Otherwise the 
sample is rejected and another is chosen in the same manner. Scattering Mechanics

The equations of neutron moderation are taken from simple "billiard-ball" mechanics. Detalls of the $f^{\text {th }}$ neutron collision with the moderator atoms are derived from the conservation of energy and momentum. Upon collision, the following scattering data are calculated sequentially to obtain the energy loss and the scattering angle.

$$
\begin{gathered}
x_{j}^{2}=\frac{1}{\left(A_{j}+1\right)^{2}}\left[u_{j}^{2}+A_{j}^{2} U_{j}^{2}+2 A_{j} u_{j} U_{j} \cos a_{j}\right] \\
v_{j}^{2}=\frac{A_{j}^{2}}{\left(A_{j}+1\right)^{2}}\left[u_{j}^{2}+U_{j}^{2}-2 u_{j} u_{j} \cos a_{j}\right] \\
E_{j+1}=x_{j}^{2}+v_{j}^{2}+2 x_{j} v_{j} \cos b_{j} \\
\cos c_{j}=\frac{1}{x_{j}\left(A_{j}+1\right)}\left[u_{j}+A_{j} U_{j} \cos a_{j}\right] \\
\cos \Psi_{j}=\cos b_{j} \cos c_{j}+\sin b_{j} \sin c_{j} \cos \phi_{j} \\
\mu_{j}=\frac{1}{u_{j} \pm 1}\left[v_{j} \cos \Psi_{j}+x_{j} \cos c_{j}\right]
\end{gathered}
$$

The flight parameters are defined as follows:

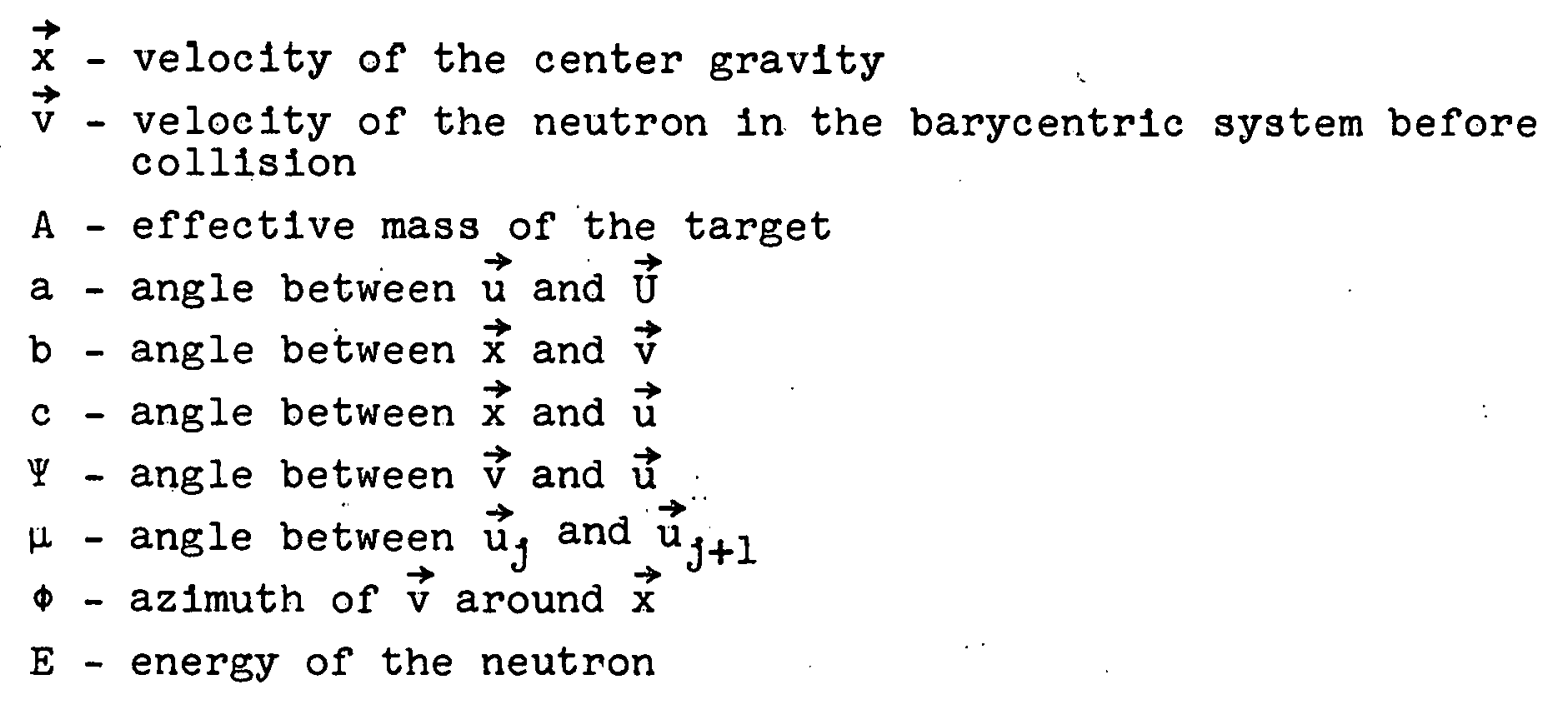


The mass of the neutron is taken as unity and the units of velocity are such that $u=\sqrt{E}$. The index $J$ is the number of the collision. The solid angle of scattering in the barycentric system (1.e., $\phi$ and $\cos$ b) is selected at random.

Accumulation of $\mathrm{M}^{2}$

The migration area, $M^{2}$, is defined as one-sixth the meansquare distance, $\overline{r^{2}}$, travelled by the neutrons in slowing down.

When the flight parameters have been obtained the contribution to the migration area is computed and the contents of the storage for $\overline{\mathrm{r}^{2}}$ are brought up to date. A general expression for the second moment of the slowing-down distribution derived by Coveyou (1956) was adapted forthe codêsthe mean-square distance travelled in the course, of $f$ collisions is

$$
\overline{r^{2}}=2 \sum_{\alpha=1}^{j} \sum_{\beta=1}^{\alpha} \lambda_{\alpha} \lambda_{B}\left(\mu_{B} \mu_{B+1} \cdots \mu_{\alpha+1}\right)
$$

The mean-free-paths, $\lambda$, are plcked from a table where they are stored as functions of energy. The quantity $\mu_{\beta}$ is the computed. cosine of the scattering angle at collision $\beta$.

The use of the general expression for $\overline{r^{2}}$ is a major factor in the speed and precision of the computation. This expression gives the proper average of all possible path lengths between successive collisions, and all possible azimuthal angles of scattering. Thus, every neutron history is a statistical representation of all neutrons that have experienced the same succession of energies of degradation. Only those spatial details of the scattering event necessary to obtain the cosine of the scattering angle, $\mu$, were required in the computation. 
The flight between collisions has now been completed and pertinent data are punched. The next event (scattering or absorption) is determined from the energy as before, and the process is repeated. A neutron history is terminated when the neutron is absorbed or when its energy falls below some cutoff level.

The speed of computing neutron historles depends upon the number of collisions required per history. A large fraction of the machine time is spent in the accumulation of the $\overline{r^{2}}$ function. The contributions of flights with large $f$ involve many more arithmetic operations than those for small $\mathrm{j}$. The computing rate $R$ in terms of the average number of collisions per neutron, $J$, is given by the empirical formula

$$
R(\text { neutrons } / \mathrm{hr})=\frac{3900}{\mathrm{~J}^{2}}+\frac{550}{\mathrm{~J}}
$$

Useful results can be obtained in a double shift of IBM 650 operation. For example the migration area from fission to $1.46 \mathrm{ev}$ in $\mathrm{H}_{2} \mathrm{O}$ was computed from 750 neutron histories in sixteen hours with a standard deviation of $0.6 \%$.

\section{RESULTS}

A. Migration Areas

The method is primarily one for computing migration areas in infinite homogeneous moderators. Diffusion constants and temperature coefficients were obtained by variation of input parameters in the migration area calculation. Components of the migration area above and below the indium resonance ( $1.46 \mathrm{ev}$ ) were obtained separately. The familiar discrepancy between computed and 
measured values of the migration area for $\mathrm{H}_{2} \mathrm{O}$ from fission to $1.46 \mathrm{ev}$ remained; the computed value was $3 \mathrm{~cm}^{2}$ below the measured value. For $\mathrm{D}_{2} \mathrm{O}$, however, the computed value was $8 \mathrm{~cm}^{2}$ above the measured value. The results are listed in Table $I$.

\section{TABLE I}

\section{CALCULATED MIGRATION AREAS}

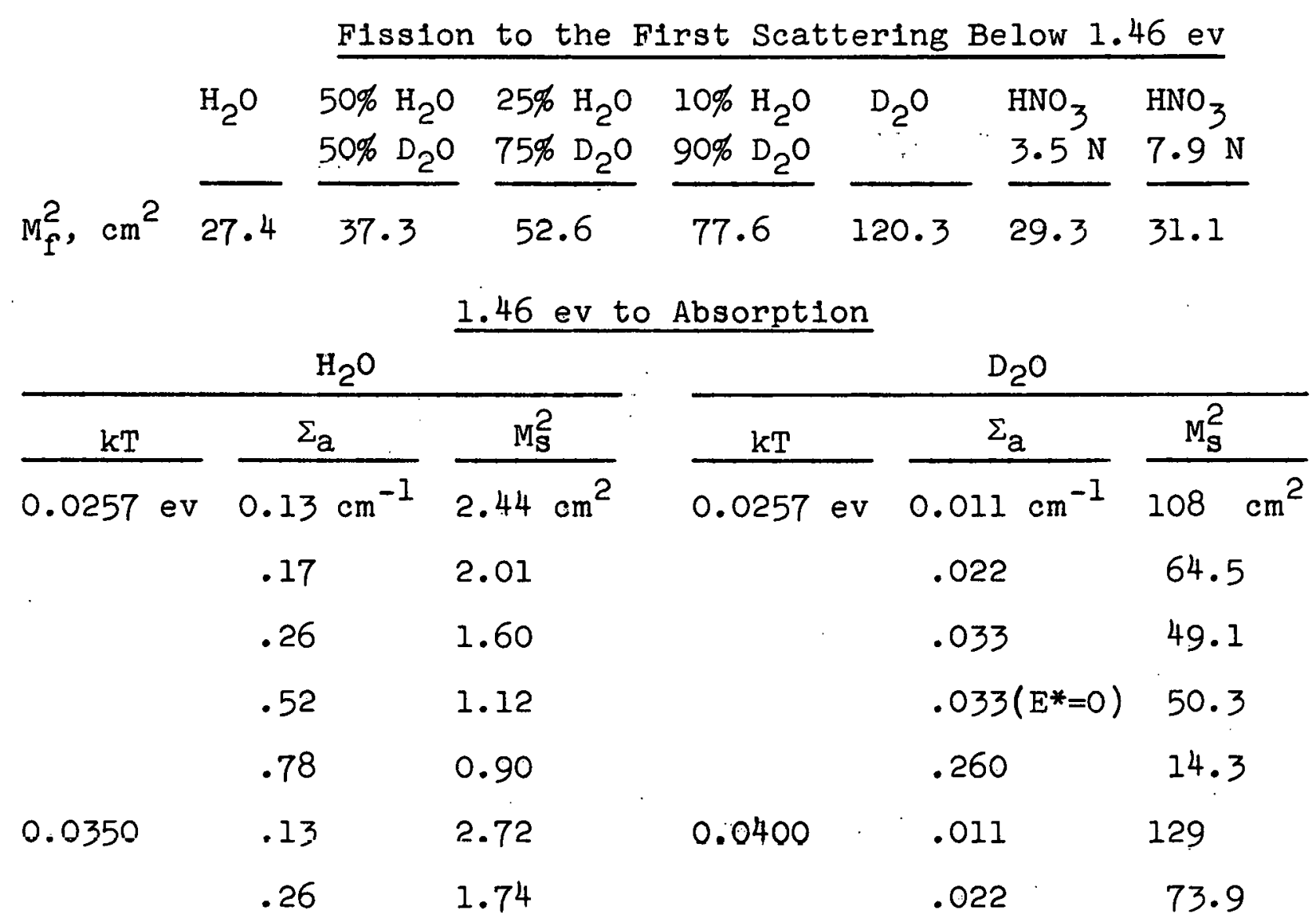

NOTES: The migration area from fission to $1.46 \mathrm{ev}$ is calculated to be $26.7 \mathrm{~cm}^{2}$ for $\mathrm{H}_{2} \mathrm{O}$ and $119.5 \mathrm{~cm}^{2}$ for $\mathrm{D}_{2} \mathrm{O}$. $\Sigma_{a}$ is defined as the maxwellian average of the $1 / v$ cross section at $25^{\circ} \mathrm{C}$. The case with $E^{*}=0$ shows the effect of neglecting chemical binding. 
The energy range for slowing down is divided at $1.46 \mathrm{ev}$, the energy of the In-115 resonance, to permit the results to be compared with experiments. In the high energy range the source of neutrons was taken to be the fission energy distribution. The system was assumed to be nonabsorbing, and the moderator atoms were assumed to be unbound and at rest. When a neutron emerged from collision with energy less than $1.46 \mathrm{ev}$, its contribution to $\mathrm{M}^{2}$ was computed and the history terminated. In the low energy range the source energy was assumed constant at $1.46 \mathrm{ev}$. The absorption rate was taken to behave as $1 / \mathrm{v}$ and was varied in strength to study the validity of the relation $\mathrm{M}^{2}=\frac{\mathrm{D}}{\Sigma_{\mathrm{a}}}+\tau$. The moderator atoms were in a maxwellian distribution and were assumed bound for collisions occurring below the threshold of vibration, $E^{*}$. Neutron histories were terminated by absorption. The fast and slow components so obtalned cannot simply be added to yleld the total migration area because slowing down in the energy "overlap" of the first collision below $1.46 \mathrm{ev}$ is computed for both components. Migration areas through the energy overlap were obtained from short runs using a $\frac{1}{E}$ distribution above $1.46 \mathrm{ev}$ as a source. Both $\mathrm{M}^{2}$ for scatterings to energles below $1.46 \mathrm{ev}$ and $\mathrm{M}^{2}$ for scatterings to energies within the interval 1.53 to $1.39 \mathrm{ev}$ were accumulated. The difference between these two concepts of $\mathrm{M}^{2}, 0.7 \mathrm{~cm}^{2}$ for $\mathrm{H}_{2} \mathrm{O}$ and $0.8 \mathrm{~cm}^{2}$ for $\mathrm{D}_{2} \mathrm{O}$, must be subtracted from the migration area to below $1.46 \mathrm{ev}$ in order to obtain the migration area to $1.46 \mathrm{ev}$.

$$
\text { 0lcott (1956) measured } 111 \pm 1 \mathrm{~cm}^{2} \text { and Wade (1957) }
$$

measured $109 \pm 3 \mathrm{~cm}^{2}$ for the migration area from fission to indium in $\mathrm{D}_{2} \mathrm{O}$ contaminated with $0.2 \% \mathrm{H}_{2} \mathrm{O}$. The value interpolated for this 
$\mathrm{D}_{2} \mathrm{O}$ purity from the Monte Carlo calculations is $118 \mathrm{~cm}^{2}$. Barkov and Mukhin (1956) measured $29.4 \pm 1.5 \mathrm{~cm}^{2}$ and H1ll, Roberts, and Fitch (1955) measured $30.8 \mathrm{~cm}^{2}$ for $\mathrm{H}_{2} \mathrm{O}$. The calculation gives $26.7 \mathrm{~cm}^{2}$. Hurwitz and Zweifel (1955) computed the migration area of $\mathrm{H}_{2} \mathrm{O}$ to be 23.6 by a moments treatment of a similar model. Including the effects of anisotropic scattering of oxygen in the calculation would bring the value of $\mathrm{H}_{2} \mathrm{O}$ into better agreement with experiments but would worsen the agreement for $\mathrm{D}_{2} \mathrm{O}$. Goldstein and Certaine (1957) have found the effect of anisotropic scattering to be small. They also show that the low value computed for $\mathrm{H}_{2} \mathrm{O}$ does not arise from possible errors in the fission spectrum. Experiments that determine migration area by indium foll activations require correction for the width of the activation resonance and the thickness of the foll. The observed activation is the average value over the width of the resonance. Corrections to convert these results to the values that would be obtained with resonances of zero width were estimated. The migration area in $\mathrm{H}_{2} \mathrm{O}$, as computed to the wide energy band 1.10 to $1.94 \mathrm{ev}$ which is embraced by the indium resonance, was $0.8 \mathrm{~cm}^{2}$ larger than that to the smaller interval 1.39 to $1.53 \mathrm{ev}$. For $D_{2} 0$ the migration area to the wide band was found to be $2.8 \mathrm{~cm}^{2}$ greater than to the narrow band. Presumably, the experimental data in the literature were not corrected for this effect.

B. Diffusion Constants

The migration area of neutrons through the thermal range of energies may be related to the absorption rate by the expression $M^{2}=\frac{D}{\Sigma_{a}}+\tau_{s}$ where $D$, the diffusion constant, depends upon the temperature and scattering properties of the moderator, $\Sigma_{a}$ is the 
effective absorption cross section, and $\tau_{s}$ is the neutron age from $1.46 \mathrm{ev}$ to thermal energy. Migration areas were computed for different absorption strengths to check the validity of the linear formula and to evaluate the magnitudes of $\tau_{\mathbf{s}}$ and $D$. An absorption cross section varying as $1 / v$ was assumed throughout.

For high values of $\Sigma_{a}$ the neutron path lengths must approach zero and a linear relation cannot be valid. However, a IInear relation does hold over a large range of $L^{2}=\frac{D}{\Sigma_{a}}$ for both $\mathrm{H}_{2} \mathrm{O}$ and $\mathrm{D}_{2} \mathrm{O}$ as shown in Figures 1 and 2 . The Iinear portions extrapolate to the component of $M^{2}$ that does not vary with changes in $\Sigma_{\mathrm{a}}$. This component is interpreted as the epithermal age; $\tau_{s}=0.77 \mathrm{~cm}^{2}$ for $\mathrm{H}_{2} \mathrm{O}$ and $\tau_{s}=20 \mathrm{~cm}^{2}$ for $\mathrm{D}_{2} \mathrm{O}$. The energy at which neutrons may be considered thermal and to which $\tau_{s}$ should be computed has been debated by Cohen (1955). Our value of $\boldsymbol{\tau}_{\mathbf{s}}$ for $\mathrm{D}_{2} \mathrm{O}$ agrees with the usual $20 \mathrm{~cm}^{2}$ derived from the assumption that the neutron becomes thermal at $\mathrm{kT}$ rather than the $6 \mathrm{~cm}^{2}$ resulting from the assumption that the neutron becomes thermal at about $15 \mathrm{kT}$. Barkov, Makarın, and Mukhın (1956) have measured $\tau_{s}$ to be $1.0 \pm 0.5$ for $\mathrm{H}_{2} \mathrm{O}$.

In order to obtain $\mathrm{D}$ from $\mathrm{L}^{2}$, we arbitrarily define $\Sigma_{a}$ to be the average cross section of the $1 / v$ absorber in a maxwellian spectrum with temperature corresponding to $\mathrm{kT}=0.025 \mathrm{ev}$, or $\sqrt{\pi} / 2$ times the $2200 \mathrm{~m} / \mathrm{sec}$ cross section. The diffusion constants are then found to be $0.19 \mathrm{~cm}$ for $\mathrm{H}_{2} \mathrm{O}$ and $0.87 \mathrm{~cm}$ for $\mathrm{D}_{2} \mathrm{O}$. From values of the transport mean-free-path given in the Reactor Handbook (1955), one finds $\mathrm{D}=\frac{\lambda t r}{3}=0.16 \mathrm{~cm}$ for $\mathrm{H}_{2} \mathrm{O}$ and $0.88 \mathrm{~cm}$ for $\mathrm{D}_{2} \mathrm{O}$. The diffusion constant increases with higher moderator temperatures at constant moderator density through the influence of the increased 
agitation of moderator atoms. The effect is reflected in the higher slopes of the curves in Figures 1 and 2. The coefficient for this effect alone was found to be $0.15 \% /{ }^{\circ} \mathrm{C}$ for $\mathrm{H}_{2} \mathrm{O}$ and $0.14 \% /{ }^{\circ} \mathrm{C}$ for $\mathrm{D}_{2} \mathrm{O}$. In addition, the usual effects of density changes and spectral hardening must be included in the over-all temperature coefficlent of $\mathrm{M}^{2}$.

C. Neutron Energy Distributions

Neutron energy spectra are obtained from the Monte Carlo code by merely accumulating the number of scatterings that occur within each energy increment. An example is graphed in Figure 3. The distribution obtained by Monte Carlo colncides with that obtained from an analytical solution (Brown 1956). The comparison with the spectrum computed by a formula of Cohen (1955) Implies that the disagreement about the epithermal age is in the definition of $\tau_{s}$ and does not extend to the spectrum.

Although the direct calculation of simple spectra is faster than the Monte Carlo calculation, it is still practical to obtain spectra from the code when it is also used for other purposes. The accumulation of a spectrum is incldental to the computation of $\mathrm{M}^{2}$ and Involves no additional computing time. For instance, at the same time that temperature coefficients of $\mathrm{M}^{2}$ are computed, the temperature coefficients of the spectra can be obtained. In some cases parametric changes are more easily encompassed by the Monte Carlo method. Unusual situations, such as the effect of thermal resonances upon the spectrum, can be surveyed without additional coding. 


\section{CONCLUSION}

The code is fast and accurate enough to find frequent application at the Savannah River Laboratory. Migration areas calculated by the Monte Carlo method are based on the same physical model that is used for the analytical methods (Hurwitz and Zweifel, 1955). The results are thus subject to similar errors such as scattering isotropy and fission source error, but useful information is obtained by normalizing to the avaliable experimental points. The code is well suited to the study of the temperature variations, the effects of mixtures, arbitrary absorption and scattering functions, and arbitrary source distributions. The treatment could be extended to finite moderators by the usual artifice of adding the leakage to the absorption rate: $\Sigma_{a}^{\text {eff }}=\Sigma_{a}+\mathrm{DB}^{2}$ where $\mathrm{B}^{2}$ is the buckling.

The author wishes to thank D. S. St. John for stimulating support and W. V. Baxter for the programming and operation of the IBM 650 code. 


\section{BIBLIOGRAPHY}

1. Barkov, I. M., Makarin, V. K., and Mukh1n, K. N. (1956)

"Measurement of Slowing Down of Neutrons in the Energy Range $1.46-0.025 \mathrm{ev}$ in Water." Atomnaya Energiya $1,3,33$ (J. Nuc 1. Energy 4, 94-102).

2. Barkov, L. M. and Mukhin, K. N. (1946) "The Slowing Down of Fission Neutrons in Water." Atomnaya Energiya 1, 3, 31 (J. Nucl. Energy 4, 91-93).

3. Brown, H. D. (1956) Neutron Energy Spectra in Water, DP-64.

4. Brown, H. D. and St. John, D. S. (1954) Neutron Energy Spectrum in $\mathrm{D}_{2} \mathrm{O}, \mathrm{DP}-33$.

5. Cohen, E. R. (1955) Some Comments on the Effects of Epithermal Neutrons in a Reactor, NAA-SR-1127.

6. Coveyou, R. R. (1956) Monte Carlo Calculation of Neutron Age in water. ORNL-2081, p 144-145.

7. Goldstein, H. and Certaine, J. (1957) Some Recent Calculations of the Age of Fission Neutrons in Water. ORNL-2389, p 174-175.

8. Hill, J. E., Roberts, I. D., and Fitch, T. E. (1955) "Slowing Down Distribution of U-235 Fission Neutrons from a Point Source in Light Water." J. Appl. Phys. 26, 8, 1013-1017.

9. Hurwitz, H. and Zweifel, P. F. (1955) "Slowing Down of Neutrons with Hydrogenous Moderators." J.Appl. Phys. 26, 8, 923-931.

10. Lehmer, D. H. (1951) "Mathematical Methods In Large Scale Computing Units." Annals of the Computation Laboratory 26, p 141, Harvard University.

11. Olcott, R. N. (1956) "Homogeneous Heavy Water Moderated Critical Assemblies." Nuclear Science and Engineering 1, 4, 327 .

12. The Reactor Handbook Vol. 1 (1955) AECD-3645.

13. Rosen, L. (1955) "Techn1ques for Measurement of Neutron Cross Sections and Energy Spectra for Sources which are Continuous in Energy and Time." Proc. Intern. Conf. Peaceful Uses of Atomic Energy, $4,97$.

14. Wade, J. W. (1957) "Neutron Age in Mixtures of Light and Heavy Water." Nuclear Sclence and Engineering (in proof).

15. Wilkins, J. E., Hellens, R. L., and Zweifel, P. E. (1956) Status of Experimental and Theoretical Information on Neutron Slowing-Down Distributions in Hydrogenous Media." Proc. Intern. Conf. Peaceful Uses of Atomic Energy 5, 62-76. 
Note to Figures 1 and 2

The plotted migration areas were obtained by Monte Carlo calculations. The straight lines are least-square fits to the linear portion of the data and show the range of validity of the relation $\mathrm{M}^{2}=\frac{D}{\Sigma_{a}}+\tau$. If $\Sigma_{a}$ is defined as the average of the $\frac{l}{\mathrm{v}}$ cross section in a maxwellian flux at $25^{\circ} \mathrm{C}, \mathrm{D}$ is found to be $0.19 \mathrm{~cm}$ for $\mathrm{H}_{2} \mathrm{O}$ and $0.87 \mathrm{~cm}$ for $\mathrm{D}_{2} \mathrm{O}$. The values of $\mathrm{kT}$ refer to moderator temperatures.

Note to Figure 3

The distribution derived by Monte Carlo coincides with the analytic solution. The dashed curve is obtained from a formula of Cohen (1955). $\Sigma_{a}=0.011 \mathrm{~cm}^{-1} \quad \mathrm{kT}=0.0257 \mathrm{ev}$ 
FIGURE I

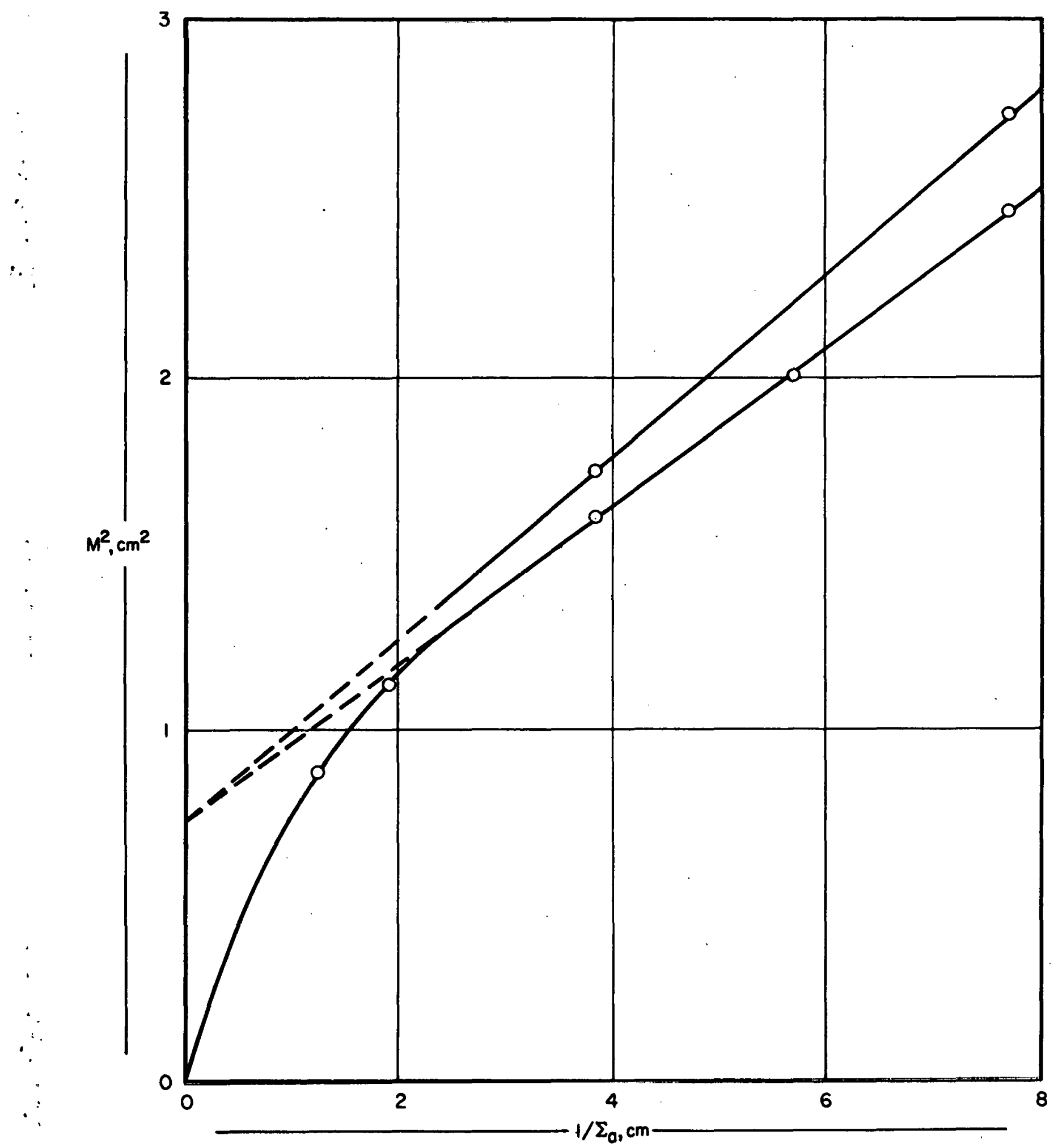

MIGRATION AREAS IN Hz FROM $1.46 \mathrm{eV}$ TO ABSORPTION

$289 \quad 019$ 
FIGURE 2

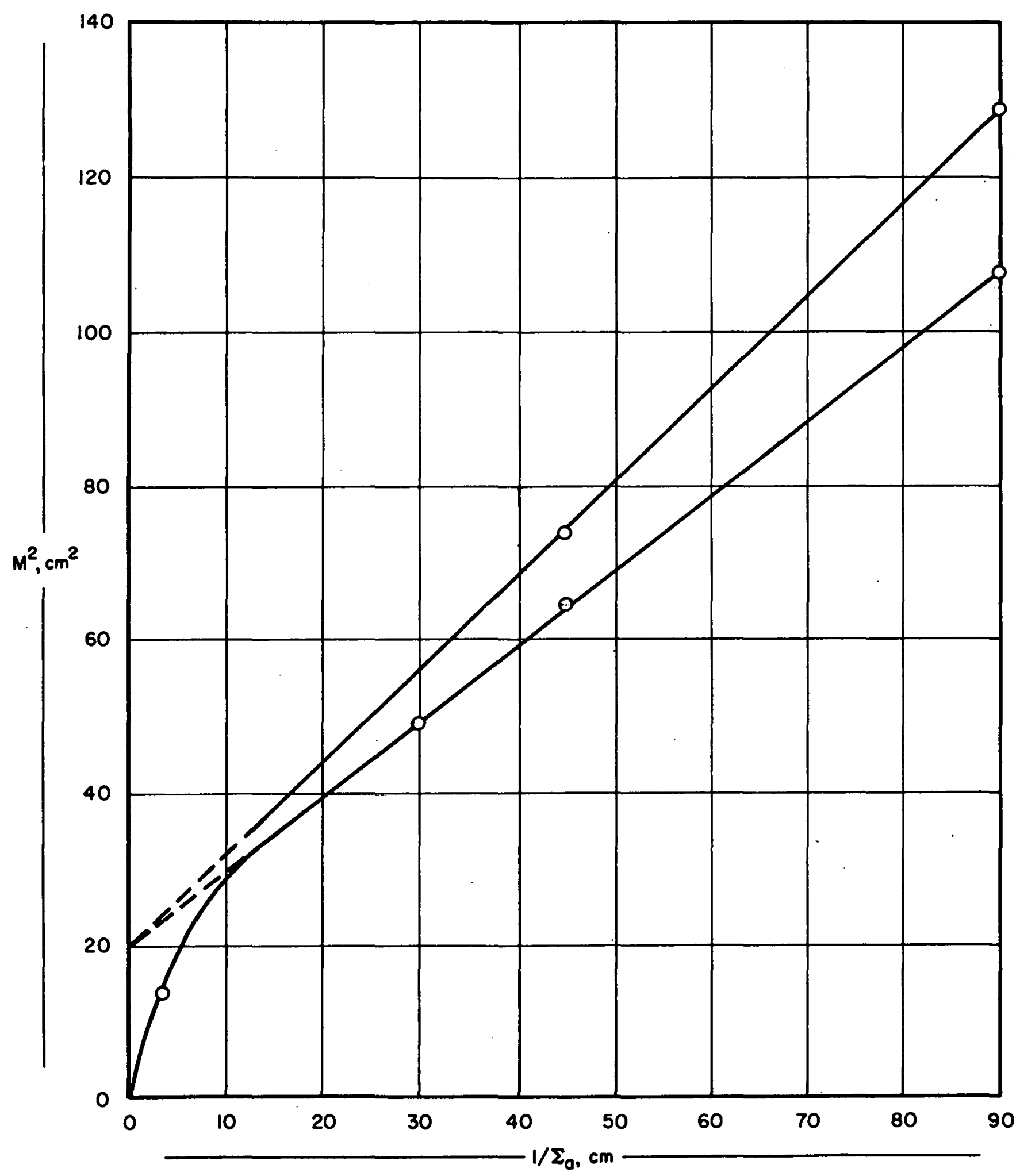

MIGRATION AREAS IN $\mathrm{D}_{2} O$ FROM 1.46 eV TO ABSORPTION 
FIGURE 3

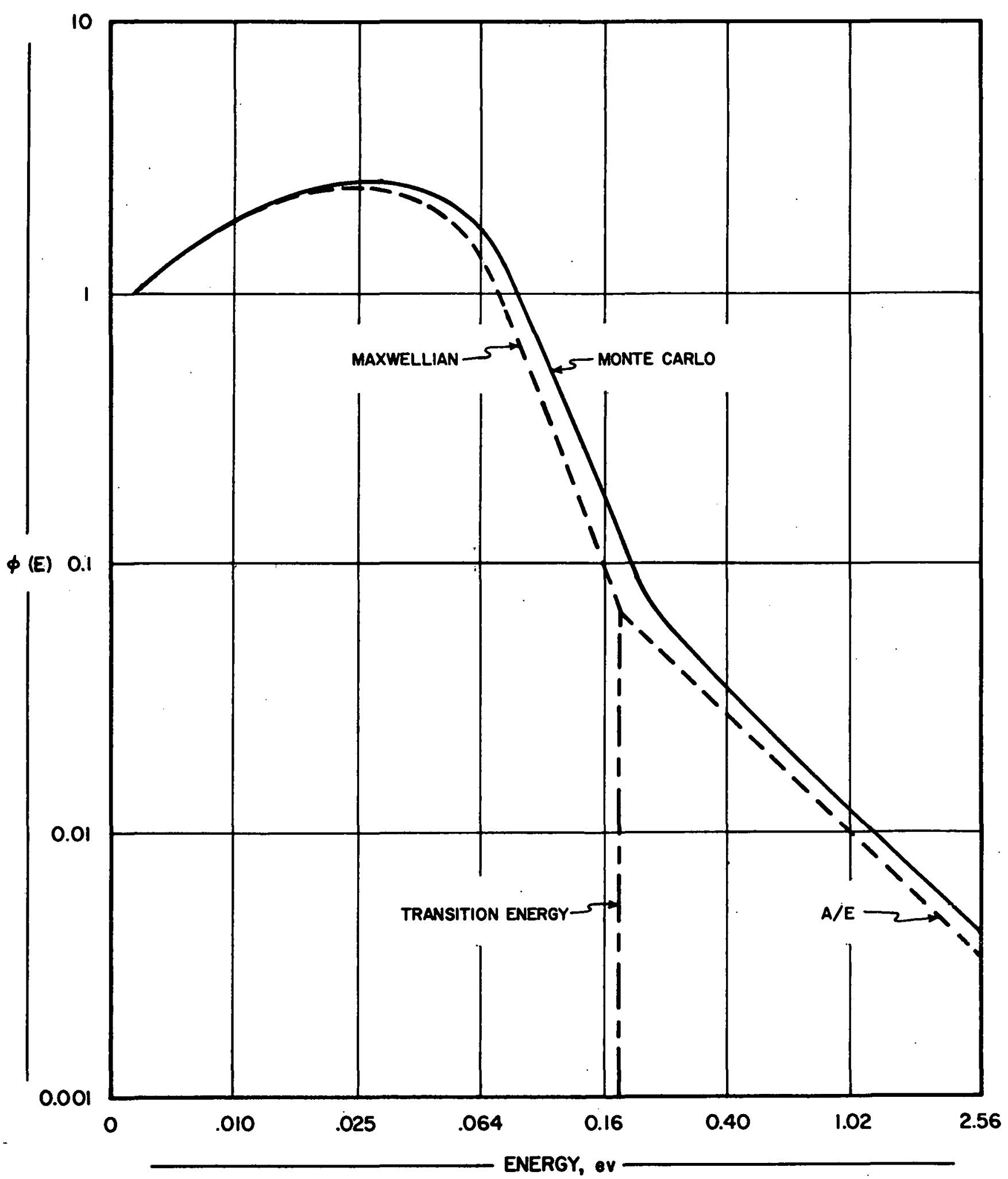

NEUTRON ENERGY DISTRIBUTION IN $\mathrm{D}_{2} \mathrm{O}$ 